\title{
BLIND EQUALIZATION OF PPM SIGNALS USING THIRD-ORDER MOMENTS
}

\author{
Patrik Pääjärvi and James P. LeBlanc
}

\author{
Luleå University of Technology \\ SE-971 87, Luleå, SWEDEN \\ \{patrik,leblanc\}@csee.ltu.se
}

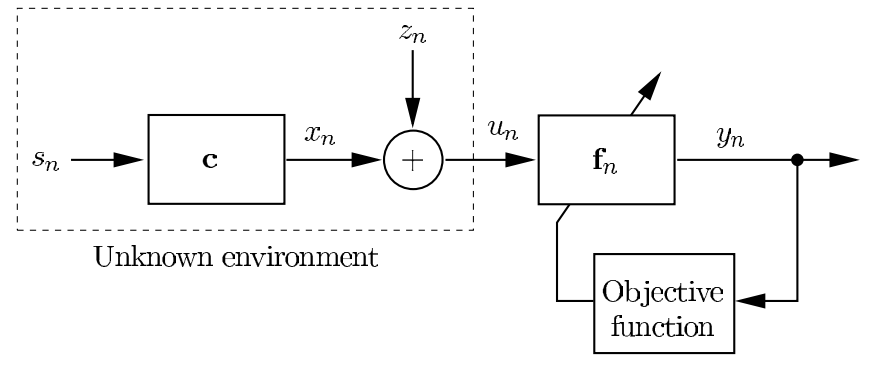

Fig. 1. Discrete-time blind adaptive equalization model.

\begin{abstract}
In pulse-position modulation (PPM) signaling, the time location of short-duration pulses are used to convey information over a communication channel. For successful noncoherent reception, the channel duration must be short compared to the symbol interval. This paper analyzes the use of third moments in a blind adaptive equalizer setting to limit the effective delay spread of the channel. Results detail the global convergence properties of the proposed method, showing that the parameters approach ISI-free settings under general conditions.
\end{abstract}

\section{INTRODUCTION}

Pulse-position modulation (PPM) signaling is based on transmitting short-duration pulses of constant magnitude, with the information being conveyed in the time location of the individual pulses. While historically mainly used in optical communication, the recent evolution of ultra-wideband (UWB) radio has spawned renewed interest in PPM as a proposed signal for wideband, carrier-free wireless communication over short range $[1,2]$.

Recent work on low-complexity noncoherent receivers for PPM show promising results in cases where the transfer rate is low compared to the maximum delay spread of the multipath channel [3]. With increasing demands for higher data rates, it becomes necessary to limit the effects of excessive channel length at the receiver. This paper proposes the use of a blind adaptive equalizer to allow noncoherent reception of high-rate PPM signals without estimating channel coefficients.

Blind adaptive equalization is used to reduce intersymbol interference (ISI) over a dispersive channel in situations where sending a training sequence is not possible. The target of adaptation is to remove enough ISI to provide a good initial setting for decision-directed (DD) equalization. With reliable estimates of the transmitted symbols, DD equalization can quickly move parameters close to the minimum mean squared error (MMSE) setting [4].

The principle of blind equalization is shown in Figure 1. The channel $\mathbf{c}$ filters the transmitted source $s$ with added noise $z$, forming the observed (received) signal $u$. Adjustment of the equalizer $\mathbf{f}_{n}$ is governed by an objective function in the form of a non-linear function of the output $y$. If the source signal is a sequence of i.i.d. samples, the objective function may be chosen from several broad classes [5]. Higher-order moments (order $>2$ ) of $y$ are often used since they are easy to estimate from instantaneous sample values. The $p^{\text {th }}$ moment of a random variable $\xi$ is defined as

$$
\mathcal{M}_{p}(\xi) \triangleq \mathrm{E}\left\{\xi^{p}\right\} .
$$

Fourth moments are a popular choice and form the basis of the widely known constant modulus algorithm (CMA) [6, 7, 8].

When $s$ is not an i.i.d. sequence, the objective function should reflect some characteristic of $s$ to be emphasized in y. Asymmetric signals, i.e. signals characterized by positive skewness (normalized third moment), allow the use of third moments in blind equalization. While having applications in various other fields $[9,10,11]$, third moments are rarely considered in digital communication since almost all modulation types yield symmetric signals with zero skewness, prohibiting the use of odd moments. PPM signals, however, are a notable exception, being asymmetric by construction. Previous studies have demonstrated that exploiting asymmetry by choosing third moments over fourth moments, when possible, generally leads to faster adaptation and improved robustness to noise [12].

While fourth-order methods, especially CMA, have been subject of much study in the past [8], the details of thirdorder methods have attracted less attention. This paper examines global convergence properties of a third-moment based blind equalization scheme under PPM signaling. It is shown that the proposed method is insensitive to white Gaussian noise, and moves the parameters of a sufficiently long equalizer close to ISI-free settings. 


\section{NOTATION AND MODEL DESCRIPTION}

\subsection{M-PPM signal}

An M-PPM signal $\varsigma$ is made up of consecutive symbol frames of $\mathrm{M}$ samples $(\mathrm{M} \geq 3)$. To each frame, one of $\mathrm{M}$ equally probable symbols $\left\{a_{k}\right\}_{k=1 \ldots M}$ is mapped by assigning unity to the $k^{\text {th }}$ sample and zero to the remaining $\mathrm{M}-1$ samples. An example with $\mathrm{M}=4$ and the three consecutive symbols $a_{2}, a_{4}$ and $a_{1}$ starting at frame $N$ would give

$$
\varsigma=\{\cdots \underbrace{01100}_{N} \underbrace{00001}_{N+1} \underbrace{10000}_{N+2} \cdots\} .
$$

The transmitted sequence $s$ is then formed by subtracting off the mean to achieve a DC-free signal, $s \triangleq \varsigma-\mathrm{E}\{\varsigma\}=\varsigma-\frac{1}{\mathrm{M}}$. The autocorrelation of $s$ is

$$
\mathrm{E}\left\{s_{n} s_{n+m}\right\}= \begin{cases}\frac{\mathrm{M}-1}{\mathrm{M}^{2}}, & m=0 \\ \frac{|m|}{\mathrm{M}^{3}}-\frac{1}{\mathrm{M}^{2}}, & |m|=1 \ldots \mathrm{M}-1 \\ 0, & |m| \geq \mathrm{M} .\end{cases}
$$

From the construction of $\varsigma$, samples within the same frame are dependent, while samples from distinct frames are independent. Hence, $s$ is correlated up to lags $\mathrm{M}-1$. The skewness of $s$ is

$$
\frac{\mathrm{E}\left\{s^{3}\right\}}{\left(\mathrm{E}\left\{s^{2}\right\}\right)^{3 / 2}}=\frac{\mathrm{M}-2}{\sqrt{\mathrm{M}-1}}
$$

which is non-zero if $\mathrm{M} \geq 3$, and increases strictly with increasing M. Restricting M to be greater than or equal to three therefore guarantees $s$ to be an asymmetric signal.

In practice, an enhanced PPM signaling format incorporating pseudo-random time-hopping would be used in multiple-access systems [1]. The simple form of $s$ will be used in this paper, since the addition of time-hopping can be approximately modeled by a larger $\mathrm{M}$. The influence of $\mathrm{M}$ on the performance of the proposed blind equalization method is investigated in Sections 4 and 5.

\subsection{Channel and equalizer}

Referring to Figure 1 , the adaptive equalizer $\mathbf{f}_{n}$ is FIR of length $N_{f}$, denoted at time $n$ by the $N_{f} \times 1$ column vector $\mathbf{f}_{n} \triangleq\left[\begin{array}{llll}f_{0 n} & f_{1 n} & \cdots & f_{\left(N_{f}-1\right) n}\end{array}\right]^{\mathrm{T}}$. The impulse response of the channel $\mathbf{c}$ is assumed to have the substantial part of its energy concentrated to the $N_{c}$ first samples. It is therefore modeled as an FIR system, represented by the $N_{c} \times 1$ vector $\mathbf{c} \triangleq\left[\begin{array}{llll}c_{0} & c_{1} & \cdots & c_{N_{c}-1}\end{array}\right]^{\mathrm{T}}$, with $c_{j}$ being the $j^{\text {th }}$ sample of the impulse response. c represents the aggregate response of the transmitter's pulse-shaping filter, the wireless channel and possible receiver filters. Also, define the $N_{f} \times N_{t}$ channel convolution matrix

$$
\mathbf{C} \triangleq\left[\begin{array}{ccccc}
c_{0} & \cdots & c_{N_{c}-1} & & \\
& \ddots & & \ddots & \\
& & c_{0} & \cdots & c_{N_{c}-1}
\end{array}\right]
$$

where $N_{t}=N_{c}+N_{f}-1$, and the $N_{t} \times 1$ combined channelequalizer response vector

$$
\mathbf{t}_{n} \triangleq \mathbf{C}^{\mathrm{T}} \mathbf{f}_{n}
$$

\section{BLIND EQUALIZATION STRATEGY}

Perfect equalization is said to hold if no ISI is present in $y$ after equalization. In practice, a sufficient requirement is

$$
y_{n} \approx \alpha s_{n-\delta}
$$

for some scale factor $\alpha$ and delay $\delta$. Adaptation may switch to decision-directed mode once enough ISI has been removed during the initial adaptation. This is the reason why strict equality is not necessary in (2).

The equalizer under investigation is iteratively adjusted to maximize the third moment of $y$ through the gradient ascent algorithm

$$
\begin{gathered}
\mathbf{f}_{n+1}=\mathbf{f}_{n}+\mu \widehat{\nabla}_{n}, \\
\mathbf{f}_{n+1} \leftarrow \frac{\mathbf{f}_{n+1}}{\left\|\mathbf{f}_{n+1}\right\|}
\end{gathered}
$$

where $\mu$ is a small stepsize, $\|\cdot\|$ is the Euclidean norm and $\widehat{\nabla}_{n}$ is some instantaneous estimate of the gradient $\nabla$ of $\mathcal{M}_{3}$ with respect to $\mathbf{f}_{n}$,

$$
\nabla \triangleq \frac{\partial \mathcal{M}_{3}(y)}{\partial \mathbf{f}_{n}}
$$

The normalization ( $3 b)$ is necessary to keep the algorithm numerically stable; since increasing the amplitude of $y$ by 'stretching' $\mathbf{f}_{n}$ increases $\mathcal{M}_{3}(y)$ without improving equalization. Without (3b), the norm of $\mathbf{f}_{n}$ grows rapidly over iterations.

\subsection{Why use $\mathcal{M}_{3}$ ?}

A necessary condition in order for the algorithm (3) to be successful in equalizing channels with M-PPM signals, is that a high value of the objective $\mathcal{M}_{3}(y)$ corresponds to low levels of ISI in $y$. While the next section will show that this is indeed the case, some rationales for using third moments are described.

- With $s$ asymmetric, the channel output $x$ will appear more symmetric if $\mathbf{c}$ is non-trivial, due to $180^{\circ}$ phase shifts from reflections. Therefore, adapting $\mathbf{f}_{n}$ to promote asymmetry in $y$ should recover $s$.

- $s$ has an impulsive appearance (i.e. its pdf has a positive heavy tail). Such impulsiveness is characterized by large positive skewness. The observed signal $u$ generally appears less impulsive (with small or zero skewness). The objective function should hence promote impulsiveness in $y$.

\subsection{Topological view of equalizer adaptation}

Due to the normalization step (3b), we can view the adaptation algorithm (3) as traversing the $\left(N_{f}-1\right)$-dimensional unit sphere in search of maximum points, i.e. points where $\nabla$ vanishes and the Hessian is negative definite. With $\mathcal{M}_{3}$ being a 
cubic function of $\mathbf{f}_{n}$, the objective becomes a multimodal surface on the sphere. Optimization over such surfaces has been widely explored for CMA (see for example [8] and references therein). Some general observations are worth mentioning.

- There is usually no single global maximum point, but several local maxima with equal ISI reduction performance. With $\mu$ small, the length and initialization of $\mathbf{f}_{n}$ determines which one is approached.

- 'False' or 'bad' local maxima may also exist where adaptation can get stuck at high ISI parameter settings. These may be avoided by choosing a 'good' initial setting of $\mathbf{f}_{n}$.

- Adaptation may get stalled near saddle points (points with zero gradient and indefinite Hessian) during adaptation.

- Additive noise can perturb the objective surface, moving maxima away from their noise-free locations.

- Compared to i.i.d. sources, temporal correlation in $s$ may move desired maxima and even give rise to new, unwanted ones. This effect has previously been observed for CMA [13].

\subsection{Convergence in combined channel-equalizer space}

Blind equalization by maximizing $\mathcal{M}_{3}(y)$ will be considered justified if (2) is obtained at maximum points. However, with the channel being unknown, generally such equalizer settings can not be formulated. Following the style of [14] and many others, we instead examine the objective surface in combined channel-equalizer space.

With $\mathbf{t}_{n} \in \mathbb{R}^{N_{t}}$, the points corresponding to zero ISI are

$$
\mathbf{t}_{n}=\mathbf{e}_{\delta} \triangleq\left[\begin{array}{lllllll}
0 & \cdots & 0 & \alpha & 0 & \cdots & 0
\end{array}\right]^{\mathrm{T}},
$$

with the non-zero $\alpha$ occurring at element $\delta$. This would give $y_{n}=\alpha s_{n-\delta}$. Perfect equalization is thus achieved if (3) converges to $\mathbf{e}_{\delta}$ for some $1 \leq \delta \leq N_{t}$. As stated earlier, (2) is usually sufficient so that convergence to a neighborhood of $\mathbf{e}_{\delta}$ will do.

It should be pointed out that; unless $\mathbf{f}_{n}$ is infinitely long or fractionally spaced (oversampled), not all of $\mathbf{t}_{n}$-space $\left(\mathbb{R}^{N_{t}}\right)$ can be reached for an arbitrary channel. So it may not be possible in practice to reach $\mathbf{e}_{\delta}$ for all values of $\delta$. However, it is commonly argued that if the equalizer is 'long enough', there are at least some $\mathbf{e}_{\delta}$ to which adaptation may converge [14].

\section{OBJECTIVE SURFACE ANALYSIS}

In this Section, the global convergence properties of the proposed blind equalization algorithm are investigated. First, it is shown that the objective function is invariant to white Gaussian noise. Second, equations for the stationary points on the objective surface are derived for arbitrary lengths of combined channel-equalizer response $\mathbf{t}_{n}$. Finally, the objective surface is visualized for a three-tap $\mathbf{t}_{n}$-vector to gain some insight into the behavior of the method.

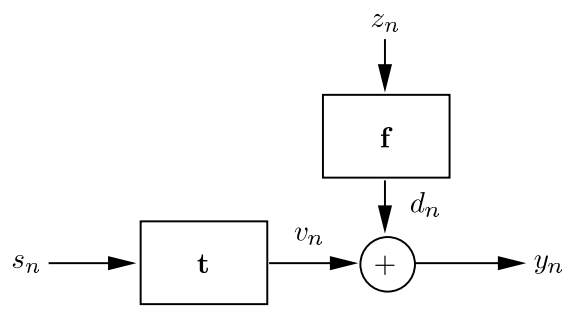

Fig. 2. Combined channel-equalizer model.

\subsection{Assumptions}

The following assumptions are made throughout the remainder.

A1) All sequences and vectors are real.

A2) The channel noise $z$ is zero-mean, white and Gaussian, independent on $s$.

A3) The adaptation stepsize $\mu$ is small, so that $\mathbf{f}_{n}$ may be regarded as approximately constant compared to all signals.

A4) The channel is assumed to be slowly varying compared to the time index $n$. Thus, $\mathbf{C}$ is also regarded as constant.

A5) $\mathbf{f}_{n}$ has infinite length.

Under Assumptions $\mathrm{A} 3$ and $\mathrm{A} 4, \mathbf{f}_{n}$ and $\mathbf{t}_{n}$ are hereafter regarded as constant vectors, denoted by $\mathbf{f}$ and $\mathbf{t}$ respectively. A5 simplifies analysis and is standard in analysis of blind systems (see for example [14]).

Keeping in mind the practical limitations on $\mathbf{f}$ mentioned in Section 3.3, the combined channel-equalizer model in Figure 2 is introduced for purpose of analysis. The noise $d$ is $z$ filtered through $\mathbf{f}$. With $z$ white and Gaussian, $d$ will be colored Gaussian noise, independent on the noise-free channel output $v$.

\subsection{Noise invariance of $\mathcal{M}_{3}$}

First, we demonstrate that the third moment is unaffected by white Gaussian noise. $\mathcal{M}_{3}(y)$ expressed in terms of $v$ and $d$ is

$$
\mathcal{M}_{3}(y)=\mathrm{E}\left\{(v+d)^{3}\right\}=\mathrm{E}\left\{v^{3}+d^{3}+3 v^{2} d+3 v d^{2}\right\} .
$$

With $v$ being zero-mean, and with $d$ zero-mean, Gaussian and independent of $v$, we have

$$
\mathcal{M}_{3}(y)=\mathrm{E}\left\{v^{3}\right\} \text {. }
$$

The objective surface is not perturbed by $z$, and therefore white Gaussian noise does not introduce any bias in gradient estimates used in the adaptation algorithm (3a).

\subsection{Stationary points for general $N_{t}$}

Equations for the stationary points of the objective surface are now derived for arbitrary lengths of $\mathbf{t}$. As seen above, the noise $d$ may be disregarded in the following.

With the combined channel-equalizer response $\mathbf{t}$ defined in (1), let $\mathbf{s}_{n} \triangleq\left[s_{n} s_{n-1} \cdots s_{n-N_{t}+1}\right]^{\mathrm{T}}$ so that $y_{n}=v_{n}=$ 
$\mathbf{t}^{\mathrm{T}} \mathbf{s}_{n}=\mathbf{f}^{\mathrm{T}} \mathbf{C} \mathbf{s}_{n}$. Expressing the third moment of $y_{n}$ in terms of $s_{n}$ yields $\mathcal{M}_{3}\left(y_{n}\right)=\mathrm{E}\left\{y_{n}^{3}\right\}=\mathrm{E}\left\{\left(\mathbf{f}^{\mathrm{T}} \mathbf{C} \mathbf{s}_{n}\right)^{3}\right\}$. The stationary points of the objective surface with respect to the equalizer parameters are found by solving

$$
\boldsymbol{\nabla}=\frac{\partial \mathcal{M}_{3}\left(y_{n}\right)}{\partial \mathbf{f}}=\mathrm{E}\left\{y_{n}^{2} \mathbf{C} \mathbf{s}_{n}\right\}=\mathbf{C E}\left\{\left(\mathbf{t}^{\mathrm{T}} \mathbf{s}_{n}\right)^{2} \mathbf{s}_{n}\right\}=\mathbf{0}
$$

where Assumption A4 ( $\mathbf{C}$ constant) has been used ${ }^{1}$. Invoking Assumption A5 in the case where the channel has no spectral nulls (i.e. an inverse exists), (4) has only the trivial solution

$$
\mathrm{E}\left\{\left(\mathbf{t}^{\mathrm{T}} \mathbf{s}_{n}\right)^{2} \mathbf{s}_{n}\right\}=\mathbf{0}
$$

This implies that there is a one-to-one correspondence between stationary points in $\mathbf{f}$-space and $\mathbf{t}$-space.

Solving (5) for the stationary points is possible since the statistics of $s$ are known. However, as pointed out in Section $3, \mathcal{M}_{3}(y)$ only attains maxima if the norm of $\mathbf{f}$ is restricted. Hence, $\|\mathbf{t}\|$ will be similarly constrained. Such a restriction causes no practical or theoretical limitations, since if $\mathbf{t}^{*}$ is a stationary point of $\mathcal{M}_{3}$, then so is $k \mathbf{t}^{*}$ for any $k \in \mathbb{R}$. We therefore solve (5) for $\mathbf{t}$ constrained to the manifold

$$
S_{N_{t}-1} \triangleq\left\{\mathbf{t} \in \mathbb{R}^{N_{t}} \mid\|\mathbf{t}\|^{2}=1\right\},
$$

i.e. the unit sphere in $\mathbb{R}^{N_{t}}$. Using the method of Lagrange multipliers, solving (5) under the constraint $\mathbf{t} \in S_{N_{t}-1}$ leads to

$$
\mathrm{E}\left\{\left(\mathbf{t}^{\mathrm{T}} \mathbf{s}_{n}\right)^{2} \mathbf{s}_{n}\right\}+2 \lambda \mathbf{t}=\mathbf{0},
$$

where $\lambda \in \mathbb{R}$ is a constant Lagrange multiplier. Expanding (6), and using the assumption of $\mathbf{t}$ being constant, leads to the following system of $N_{t}$ non-linear equations in the $N_{t}$ parameters $\left\{t_{m}\right\}_{m=0 \ldots N_{t}-1}$ :

$$
\sum_{i=0}^{N_{t}-1} \mathcal{R}(0, i-m) t_{i}^{2}+\sum_{\substack{i, j=0 \\ j \neq i}}^{N_{t}-1} \mathcal{R}(i-j, i-m) t_{i} t_{j}+2 \lambda t_{m}=0,
$$

where $\mathcal{R}(p, q) \triangleq \mathrm{E}\left\{s_{n} s_{n+p} s_{n+q}\right\}$ are generalized third moments of $s$. With $s$ being an M-PPM signal, these moments are functions of $\mathrm{M}$.

While solving (7) analytically is difficult (even for moderate values of $N_{t}$ ); in the limit of large $\mathrm{M}, \mathcal{R}(0,0)$ becomes the dominating monomial coefficient and (7) approaches

$$
\mathcal{R}(0,0) t_{m}^{2}+2 \lambda t_{m}=0,
$$

for $m=0 \ldots N_{t}-1$. Since all $N_{t}$ equations of the system (8) share the same monomial coefficients, finding all $2^{N_{t}+1}-2$ solutions for $\mathbf{t} \in S_{N_{t}-1}$ is straightforward. Specifically, the $N_{t}$ points $\mathbf{e}_{\delta}$ of perfect equalization are indeed solutions.

Although no $\mathbf{e}_{\delta}$ is a solution to the 'true' system (7), the approximation for large $\mathrm{M}$ suggests that stationary points exist in neighborhoods of the ISI-free points. This is confirmed in an experiment in Section 5 along with the following lowdimensional example.

\footnotetext{
${ }^{1} \mathrm{~A}$ constant scale factor not affecting the results has been left out for notational simplicity.
}

Table 1. Stationary points $\left(t_{0} t_{1} t_{2}\right)$ on $S_{2}$ for large M.

\begin{tabular}{c|c|c|c} 
Class & Solution subset & Type & Legend \\
\hline$C_{1}^{+}$ & $\{(100)(010)(001)\}$ & Max & $*$ \\
$C_{2}^{+}$ & $\frac{1}{\sqrt{2}}\left\{\left(\begin{array}{lll}1 & 1 & 0\end{array}\right)\left(\begin{array}{lll}1 & 0 & 1\end{array}\right)(011)\right\}$ & Saddle & $\triangle$ \\
$C_{3}^{+}$ & $\frac{1}{\sqrt{3}}\left\{\left(\begin{array}{lll}1 & 1 & 1\end{array}\right)\right\}$ & Min & $\circ$ \\
$C_{1}^{-}$ & $\{(-100)(0-10)(00-1)\}$ & Min & $\bullet$ \\
$C_{2}^{-}$ & $\frac{1}{\sqrt{2}}\{(-1-10)(-10-1)(0-1-1)\}$ & Saddle & $\nabla$ \\
$C_{3}^{-}$ & $\frac{1}{\sqrt{3}}\left\{\left(\begin{array}{lll}-1 & -1 & -1\end{array}\right)\right\}$ & Max & $\star$
\end{tabular}

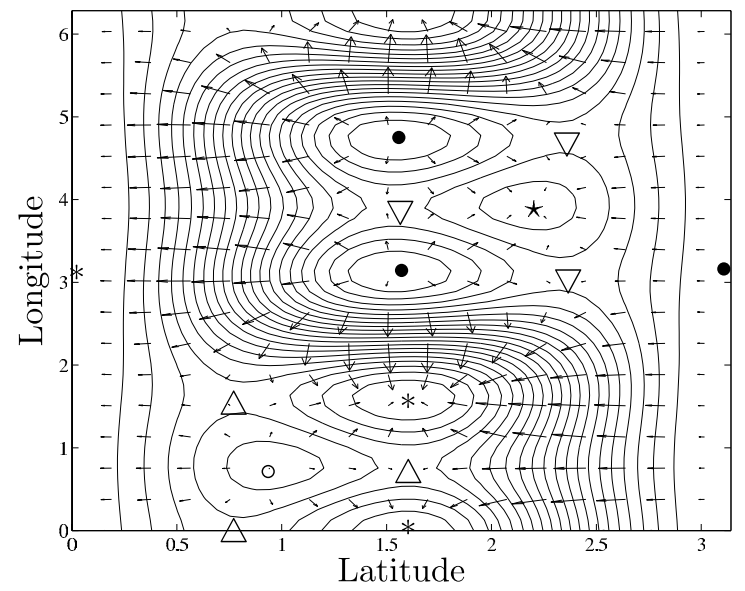

Fig. 3. Objective surface on $S_{2}$ for $\mathrm{M}=32$. Stationary points are marked by class membership according to Table 1 .

\subsection{Stationary points for $N_{t}=3$}

An improved understanding of the algorithm can be gained by visualizing the objective surface for $N_{t}=3$ with $\mathbf{t}$ on the two-dimensional unit sphere $S_{2}$. The $2^{4}-2=14$ solutions to the approximated system (8) are divided into six disjoint classes $\left\{C_{N}^{+}, C_{N}^{-}\right\}_{N=1,2,3}$, where $C_{N}^{+}\left(C_{N}^{-}\right)$is the subset of solutions with $N$ positive (negative) non-zero taps. The stationary points are listed in Table 1 with indicated type (max / min / saddle). Also in the table is a legend connecting the points to Figure 3, showing a contour plot of the objective surface on $S_{2}$ for $\mathrm{M}=32$ along with gradient arrows to illustrate its curvature. From the contour plot, the following observations are made:

- The approximated (in the limit of large M) stationary points marked in the plot all lie very close to the true ones.

- The points of perfect equalization $(*)$ are global maxima.

- A single 'bad' local maximum point $(\star)$ corresponding to severe ISI exists.

This low-dimensional example indicates that equalization of M-PPM signals by maximizing $\mathcal{M}_{3}$ does indeed lead to ISI reduction. However, the existence of the 'bad' local maxima $(\star)$ shows that adaptation could converge to a point of very poor performance, which places emphasis on initialization of the equalizer or misconvergence detection capabilities. 


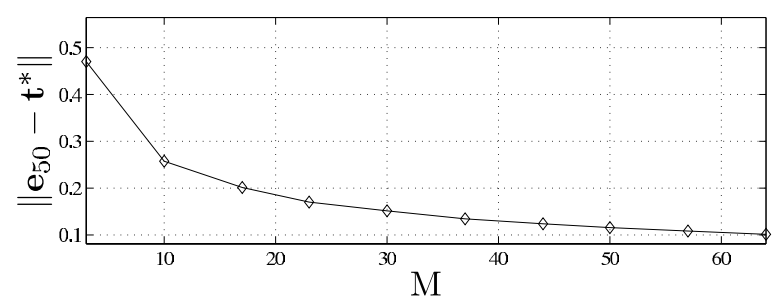

Fig. 4. Euclidean distance from $\mathbf{e}_{50}$ to nearest local maxima $\mathbf{t}^{*}$ versus $\mathrm{M}$.

\section{NUMERICAL EXPERIMENT}

A numerical experiment was done to support the results from Section 4 suggesting that local maxima of the proposed method coincide with the points of perfect equalization $\mathbf{t}=\mathbf{e}_{\delta}$ in the limit of large $\mathrm{M}$.

M-PPM signals for different values of $M$ between 3 and 64 were generated. For each signal, a gradient ascent over a 100-tap $\mathbf{t}$-vector was performed using a small stepsize of $\mu=5 \times 10^{-4}$, starting at $\mathbf{t}=\mathbf{e}_{50}$. After $10^{6}$ iterations (enough for all trajectories to reach convergence), the $\mathrm{Eu}-$ clidean distance from the starting point to the estimated convergence point (obtained by averaging over the final $10^{4} \mathbf{t}$ vectors) was calculated. This provides a measure of how far $\mathbf{t}=\mathbf{e}_{50}$ is from the nearest local maxima for different $\mathbf{M}$.

The result is shown in Figure 4, where the distance from a point of perfect equalization to the nearest local maxima decreases as $\mathrm{M}$ increases.

\section{CONCLUSIONS}

This paper shows the usefulness of third-order moments in blind adaptive equalization with M-PPM signaling, by demonstrating that attraction points exist close to zero-ISI settings. The invariance to white Gaussian noise was shown, and the proximity of convergence points to perfect equalization points was demonstrated in a numerical experiment. The existence of undesired (or 'false') local maxima has also been noted, which indicates a need for an initialization or problem detection strategy.

\section{REFERENCES}

[1] Moe Z. Win and Robert A. Scholtz, "Ultra-wide bandwidth time-hopping spread-spectrum impulse radio for wireless multiple access communications," IEEE Trans. Commun., vol. 48, no. 4, pp. 679-691, Apr. 2000.

[2] Liuqing Yang and Georgios B. Giannakis, "Ultrawideband communications: An idea whose time has come," IEEE Signal Processing Mag., vol. 21, no. 6, pp. 26-54, Nov. 2004.

[3] Cecilia Carbonelli and Umberto Mengali, "M-PPM noncoherent receivers for UWB applications," IEEE Trans. Wireless Commun., vol. 5, no. 8, pp. 2285-2294, Aug. 2006.
[4] Bernard Widrow and Samuel D. Stearns, Adaptive Signal Processing, Prentice-Hall, Englewood Cliffs, New Jersey, 1985.

[5] David L. Donoho, "On minimum entropy deconvolution," in Applied Time Series Analysis, D. F. Findley, Ed. Academic Press, New York, 1981.

[6] Dominique N. Godard, "Self-recovering equalization and carrier tracking in two-dimensional data communication systems," IEEE Trans. Commun., vol. COM-28, no. 11, pp. 1867-1875, Nov. 1980.

[7] John R. Treichler and Brian G. Agee, "A new approach to multipath correction of constant modulus signals," IEEE Trans. Acoust., Speech, Signal Processing, vol. ASSP-31, no. 2, pp. 459-472, Apr. 1983.

[8] C. Richard Johnson, Jr., Philip Schniter, Thomas J. Endres, James D. Behm, Donald R. Brown, and Raúl A. Casas, "Blind equalization using the constant modulus criterion: A review," Proc. IEEE, vol. 86, no. 10, pp. 1927-1950, Oct. 1998.

[9] Michael K. Broadhead, Lisa A. Pflug, and Robert L. Field, "Use of higher order statistics in source signature estimation," J. Acoust. Soc. Am., vol. 107, no. 5, pp. 2576-2585, May 2000.

[10] J. Y. Lee and A. K. Nandi, "Blind deconvolution of impacting signals using higher-order statistics," Mechanical Systems and Signal Processing, vol. 12, no. 2, pp. 357-371, Mar. 1998.

[11] A. K. Nandi, D. Mämpel, and B. Roscher, "Blind deconvolution of ultrasonic signals in nondestructive testing applications," IEEE Trans. Signal Processing, vol. 45, no. 5, pp. 1382-1390, May 1997.

[12] Patrik Pääjärvi and James P. LeBlanc, "Online adaptive blind deconvolution based on third-order moments," IEEE Signal Processing Lett., vol. 12, pp. 863-866, Dec. 2005.

[13] James P. LeBlanc, Inbar Fijalkow, and C. Richard Johnson, Jr., "CMA fractionally spaced equalizers: Stationary points and stability under IID and temporally correlated sources," International Journal of Adaptive Control and Signal Processing, , no. 12, 1998.

[14] Albert Benveniste, Maurice Goursat, and Gabriel Ruget, "Robust identification of a nonminimum phase system: Blind deconvolution of a linear equalizer in data communications," IEEE Trans. Automat. Contr., vol. AC-25, no. 3, pp. 385-399, June 1980. 